\title{
The Negative Association of Obesity with Subjective Well-Being: Is it All About Health?
}

\author{
Petri Böckerman • Edvard Johansson • Samuli I. Saarni • \\ Suoma E. Saarni
}

Published online: 26 June 2013

(C) Springer Science+Business Media Dordrecht 2013

\begin{abstract}
In this paper we examine whether health status and functional capacity explain the fact that obese individuals report significantly lower levels of subjective well-being. We use comprehensive measures of body composition such as waist circumference, body fat percentage, and lean body mass along with body mass index. All these are measured by health professionals in a population-based sample. When controlling for health and functional status, we find very limited evidence for any independent influence of obesity on subjective well-being. This implies that the adverse effects of obesity on health and functioning are the primary explanation for the observed negative relationship between obesity and subjective well-being.
\end{abstract}

Keywords Subjective well-being $\cdot$ Obesity $\cdot$ Body composition

P. Böckerman $(\bowtie)$

Labour Institute for Economic Research, Pitkänsillanranta 3A, 00530 Helsinki, Finland e-mail: petri.bockerman@labour.fi

E. Johansson

Åland University of Applied Sciences, Mariehamn, Finland

e-mail: edvard.johansson@ha.ax

S. I. Saarni · S. E. Saarni

Department of Mental Health and Substance Abuse Services, National Institute for Health and Welfare,

Helsinki, Finland

e-mail: samuli.saarni@helsinki.fi

S. E. Saarni

e-mail: suoma.saarni@helsinki.fi

S. I. Saarni

Department of Psychiatry, Helsinki University Central Hospital and University of Helsinki,

Helsinki, Finland 


\section{Introduction}

One of the most striking global health trends is the rapidly increasing prevalence of obesity (WHO 2000). More than two-thirds of the US population are overweight and more than $35 \%$ are obese $(\mathrm{BMI} \geq 30$ ) (Flegal et al. 2012). Obesity is associated with increased morbidity and mortality (Flegal et al. 2005) and higher Medicare costs (Cai et al. 2010). This trend is not limited to the USA (e.g. Audretsch and DiOrio 2007; OECD 2010). The prevalence of obesity has increased rapidly among both men and women also in Finland during the past few decades. More than $20 \%$ of the Finnish population was obese in 2001 (Lahti et al. 2010). But the increase in obesity in Finland has not been as rapid as in the USA. Despite this, the share of obese adults is higher in Finland than in other Nordic countries (Audretsch and DiOrio 2007). Thus, research on the impacts of the obesity epidemic is vital. One relatively neglected topic in the literature is the relationship of obesity with subjective well-being (SWB).

In psychology and economics, the pursuit of determining what makes individuals happy or satisfied with their lives has been carried out by means of quantitative research, using representative survey data sets with answers to questions on SWB (e.g. Oswald 1997). This research has documented several stylized facts that relate SWB to individual characteristics such as age and labour market status, among other things (e.g. Oswald 2010). However, only a few empirical studies have explored the effect of obesity on SWB in economics (Oswald and Powdthavee 2007; Stutzer 2007; Clark and Etilé 2009; Katsaiti 2012; Foster and Moore 2012). ${ }^{1}$ These studies suggest that SWB is significantly negatively affected by obesity after taking into account the effect of all other relevant individual-level covariates such as age and education.

But there is a large literature on obesity and quality of life in other fields of research (e.g. Kushner and Foster 2000; Kolotkin et al. 2001). It has been clearly documented that obese persons experience significant impairments in the quality of life as a result of their obesity. With this paper we contribute to the intersection of these two largely separate literatures.

Our paper broadens the existing knowledge on two important frontiers. Firstly, we estimate the effect of obesity on SWB conditional on functional capacity and health status. The empirical strategy allows us to detect whether obesity has a statistically significant negative effect on SWB beyond its obvious impact on health status and functional capacity (e.g. Larsson et al. 2002). Secondly, we add to the understanding of the effect of obesity on SWB by using several different measures of obesity. The sparse literature on obesity and SWB in economics and psychology has used only body mass index (BMI) as the measure of obesity. ${ }^{2}$ But there are several different measures of obesity along with BMI. This extension of the existing knowledge is particularly important, because BMI alone is not considered to be an adequate measure of obesity nor a sufficient predictor of obesityrelated health outcomes (Pischon et al. 2008; Petursson et al. 2011). The main reason for this is that BMI blurs the distinction between fat and fat-free mass such as muscle and bone (Burkhauser and Cawley 2008; Johansson et al. 2009; Wada and Tekin 2010; Bozoyan and Wolbring 2011). In this paper we use a comprehensive set of measures of body composition along with BMI. Furthermore, previous studies have used self-reported height and weight to calculate BMI. Self-reported measures may contain a substantial amount of

\footnotetext{
${ }^{1}$ Clark and Etilé (2009) observe that the negative well-being impact of individual's own BMI is lower when the individual's partner is heavier. This pattern is consistent with social contagion effects in weight.

${ }^{2}$ Body mass index is calculated as a person's weight in kilograms divided by height in metres squared.
} 
measurement error that tends to attenuate the estimated coefficients. In contrast, all measures of obesity that we use in our empirical specifications originate from professional health examinations conducted at local health centres. This implies that the measures of obesity contain a minimal amount of measurement error, which improves the precision of the estimates. This is important because the data sets that contain information on SWB and obesity are normally not particularly large.

\section{Data}

The study is based on the Health 2000 Survey, which comprehensively represents the Finnish population aged 30 years and over. ${ }^{3}$ The methods and base results of the survey have been previously described in detail (Heistaro 2008), and they are available at http://www.terveys2000.fi/. Briefly, the survey had a two-stage, stratified cluster sampling design, with double sampling of people over 80 years of age $(n=8,028)$ (Aromaa et al. 2004). Training sessions for Statistics Finland interviewers and a three-week training course for the health examination personnel were organised in August 2000 (Heistaro 2008, p. 16). Data were collected between August 2000 and July 2001. Of the original sample of 8,028 people, $93 \%$ participated in at least one part of the study.

To obtain relatively homogeneous samples, and because the adverse effects of obesity are more interesting for those who are strongly attached to the labour market, we have limited the focus to wage and salary earners aged between 30 and 54 who have a weekly working time of at least $29 \mathrm{~h}(n=2,245)$. The focus on employed persons implies that we use a sample of people who are likely to be well-functioning. This should decrease the risk of confounding by socioeconomic status and serious illnesses. The focus on full-time workers is not particularly important in the Finnish context, because part-time work is very rare in Finland compared with almost all OECD countries. The share of part-time workers was $12 \%$ in 2000, according to the Labour Force Survey by Statistics Finland.

The data set contains information on individual fat mass measurements obtained from an eight-polar bioelectrical impedance analysis, which is performed by running a small constant current through the body (Scharfetter et al. 2004). Resistance, or impedance, is higher in fat than in other types of tissue, which makes it possible to calculate the proportion of fat mass in the body. One advantage of the data is that it contains information not only on fat mass but also on other measures of obesity.

The dependent variable of the regression models that we estimate is SWB. ${ }^{4} \mathrm{SWB}$ is measured in the Health 2000 Survey by means of the following question: "All things considered, how satisfied have you been with your life as a whole during the past 30 days?" on a scale from 0 to 10 , where 0 is anchored as the poorest possible and 10 as

\footnotetext{
3 Our data contain older persons than the US data sets such as National Longitudinal Survey of Youth (NLSY) or National Health and Nutrition Examination Survey (NHANES) (see Burkhauser and Cawley 2008) that have been used in the literature on obesity.

4 SWB is a term that includes several different components, and these components are somewhat independent (e.g. Diener et al. 1999; Dolan et al. 2011; Tinkler and Hicks 2011). The Health 2000 Survey contains only one measure of SWB that we use in this paper. Thus, we use a single-item measure of SWB. There are also multi-item scales of SWB. The most commonly used of these are the Positive and Negative Affect Schedule (PANAS) scales (Watson et al. 1988) and the Satisfaction With Life Scale (SWLS) (Diener et al. 1985).
} 
the best possible quality of life. ${ }^{5}$ This is the type of survey information that is very widely used in both psychology and economics to capture SWB (e.g. Oswald 2010).

The explanatory variable of interest in the empirical specifications is obesity. In this paper, we use waist circumference, body fat percentage, lean body mass, ${ }^{6}$ waist-hip ratio, and waist-height ratio along with BMI. BMI is categorized using the WHO (World Health Organization) criteria to underweight $=$ BMI under 18.5 , normal weight $=18.5-25$, overweight $=25-30$ and obesity $=$ over 30 (WHO 2000). The individual-level control variables are self-reported. Annual individual wage data originating from the Finnish tax authorities have also been linked to the Health 2000 data set, by means of the personal identification number that every person residing in Finland has.

To measure health status and functional capacity we use the $15 \mathrm{D}$. The $15 \mathrm{D}$, available at www.15d-instrument.net, includes 15 dimensions: mobility, vision, hearing, breathing, sleeping, eating, speech, elimination, usual activities, mental function, discomfort and symptoms, depression, distress, vitality, and sexual activity (Sintonen 2001). As there is no gold standard for measuring health and functional capacity, we have also included another widely used measure, the EQ-5D (The EuroQoL Group 1990), to check the robustness of the $15 \mathrm{D}$ findings. Higher values of $15 \mathrm{D}$ and EQ-5D imply better health status.

Table 1 reports some descriptive statistics. The level of SWB is somewhat higher for women compared with men. This corresponds to the stylized facts of the literature on SWB (e.g. Oswald 2010). For both men and women the average value of BMI exceeds 25. The mean level of the $15 \mathrm{D}$ score is identical for both genders.

\section{Empirical Specifications}

We first estimate the OLS specifications with the following structure:

$$
\mathrm{SWB}=\alpha \text { Obesity }+ \text { controls }
$$

where the dependent variable is subjective well-being. The parameter of interest is $\alpha$, which captures the potential negative effect of the obesity measure under consideration on SWB. The control variables include age and its square, three education groups and annual earnings that constitute the standard control variables which have been used in the literature on SWB (e.g. Oswald 2010). The estimation results of Eq. (1) reveal whether there is an overall negative relationship between obesity and SWB. We use OLS to obtain easier quantitative interpretation for the estimated effects.

Then we proceed to estimate OLS models with the following structure:

$$
\mathrm{SWB}=\beta \text { Obesity }+ \text { controls }+15 \mathrm{D}
$$

Equation (2) has an otherwise similar structure as Eq. (1), but these models also control for health status and functional capacity by adding $15 \mathrm{D}$ to the set of explanatory variables. Thus, the estimates of Eq. (2) reveal whether SWB is negatively affected by obesity conditional on health status and functional capacity. If the remaining effect of obesity $(\beta)$ on SWB is negative and statistically significant, we obtain prima facie evidence that obesity affects SWB not only through health and functional capacity. The other potential channels of influence include, for example, social stigma associated with obesity.

\footnotetext{
5 SWB is likely to be relatively stable over short periods of time, in a general population sample like ours.

${ }^{6}$ Lean body mass consists of muscles and bones.
} 
Table 1 The mean values and standard deviations of the variables
SD are reported in parentheses. BMI classifications: underweight (BMI $\leq 18.5)$, normal weight $(18.5<\mathrm{BMI} \leq 24.99)$, overweight $(25 \leq \mathrm{BMI} \leq 29.99)$, obese $(\mathrm{BMI} \geq 30)$. Shares may not add up owing to rounding

\begin{tabular}{lll}
\hline & $(1)$ & $(2)$ \\
& Women & Men \\
\hline SWB (0-10) & $8.20(1.15)$ & $8.10(1.31)$ \\
BMI & $25.59(4.78)$ & $26.59(3.88)$ \\
Underweight (shares) & $0.01(0.10)$ & $0.00(0.05)$ \\
Normal weight (shares) & $0.52(0.52)$ & $0.36(0.48)$ \\
Overweight (shares) & $0.30(0.46)$ & $0.47(0.50)$ \\
Obese (shares) & $0.16(0.37)$ & $0.17(0.37)$ \\
Waist circumference $(\mathrm{cm})$ & $84.34(12.21)$ & $95.65(10.88)$ \\
Body fat percentage $(\%)$ & $29.92(7.06)$ & $20.35(5.84)$ \\
Lean body mass $(\mathrm{kg})$ & $47.83(5.75)$ & $66.86(8.38)$ \\
Waist-hip ratio & $0.84(0.06)$ & $0.96(0.06)$ \\
Waist-height ratio & $0.51(0.08)$ & $0.54(0.06)$ \\
15D score & $0.95(0.05)$ & $0.95(0.06)$ \\
N & 1,134 & 1,111 \\
\hline
\end{tabular}

However, if the estimate of $\beta$ is not significant, but if in Eq. (1) the parameter $\alpha$ is negative and significant, the results point out that the total adverse effect of obesity on SWB is driven by the fact that obesity considerably decreases a person's health status and subsequent functional capacity.

These specifications are estimated by means of several different measures of obesity, as noted earlier. We estimate the models for men and women separately, because the negative effect of obesity on SWB may differ between men and women. For example, several earlier studies report that the negative wage effects of obesity are substantially larger for women (Cawley 2007; Cawley and Ruhm 2012).

The baseline results use OLS. They constitute descriptive regressions and reveal essentially conditional correlations between the variables of interest. An alternative to OLS to control for bias on observables is the semi-parametric statistical matching approach, propensity score matching (PSM) (Heckman et al. 1998), which compares subjective wellbeing outcomes for individuals exposed to obesity with 'matched' non-obese individuals. Matching has important advantages over regression-based methods. Being a non-parametric method, matching does not impose specific linearity assumptions on the evaluated effects. Furthermore, matching explicitly tries to find for each untreated unit a similar treated unit to evaluate the counterfactual, i.e. what would happen to the treatment group without the treatment. PSM has been used previously to address various issues of health. For example, Böckerman and Ilmakunnas (2009) use various matching methods to examine the effect of unemployment on self-assessed health.

We estimate the propensity to be exposed to obesity (having a BMI score of 30 or more) with a probit model incorporating the individual-level control variables, following Morris (2007). These are the same as the controls in the OLS models. PSM enables us to recover the average treatment effect for the treated (ATT) as well as the average treatment effect for the untreated (ATU). The weighted sum of the two is the average treatment effect (ATE), namely the impact that obesity has on the SWB of a randomly chosen individual. We construct the counterfactuals from those who are not obese (i.e. have a BMI of less than 30). Because we have a relatively small sample, we use a Kernel estimator, which identifies the counterfactual outcome as a weighted average of the outcomes for non- 
treated cases within the caliper where the weight given to non-treated cases is in proportion to the closeness of the comparator case to the treated case.

\section{Results}

\subsection{Baseline Estimates}

The category for obese individuals obtains a significant negative coefficient for both women and men without controlling for 15D (Table 2, Columns 1 and 3). The quantitative magnitude of the estimates is substantial. For example, the level of SWB is 0.34 points lower on a scale of $0-10$ for those men who are obese, other things being equal. For women the difference is somewhat smaller, being 0.20 points. $^{7}$ Then we proceed to add $15 \mathrm{D}$ to the set of controls. This has a considerable impact on the relationship between obesity and SWB. As expected, those who are healthier and have a better functional capacity also have a much higher level of SWB. The most important finding is that the category for obese individuals no longer obtains a statistically significant negative coefficient (Table 2, Columns 2 and 4). This implies that the adverse health effects of obesity fully explain the negative relationship between obesity and SWB in Columns 1 and 3 of Table 2. Thus, obesity does not decrease SWB at the given level of health.

\subsection{Robustness Checks}

Because categorized BMI alone is not enough to measure obesity adequately, we use information on the detailed measures of body composition. The basic pattern that was revealed by using BMI remains the same when we measure obesity by using waist circumference (Table 3), body fat percentage (Table 4), waist-hip ratio (not reported) or waist-height ratio (not reported). Therefore, the findings for BMI are generally robust to different measures of obesity. However, the measure for lean body mass (total body mass-fat mass) reveals some interesting additional results (Table 5). For men we find that lean body mass is not associated with SWB, not even without controlling for 15D (Table 5, Column 3). ${ }^{8}$ However, for women we find that lean body mass is associated with a decrease in the level of SWB without a control for 15D (Table 5, Column 1). The quantitative magnitude of the associations between the measures of body composition and SWB are generally relatively small (even when not adjusted for 15D). For example, one-standard deviation change in waist circumference decreases SWB by $\sim 0.1$ points for women. (The standard deviations are reported in Table 1.)

We have also used BMI as a continuous variable. The conclusions remain generally similar to the ones in Table 2 (not reported). However, BMI is negative and statistically significant at the $10 \%$ level for men even after adding 15D to the set of controls.

Because there are different measures of individual health status and functional capacity, we have studied the robustness of the main estimation results by using EQ-5D, which is another widely used measure of health status (Garratt et al. 2002). EQ-5D, available at www.euroqol.org, has five dimensions: mobility, self-care, usual activities, pain or discomfort, and anxiety or depression. These are each divided into three categories of severity

\footnotetext{
${ }^{7}$ Linna et al. (2013) find an inverse U-shaped relationship between SWB and BMI for a sample of young Finnish men. They also report that there is no overall association between BMI and SWB in women.

8 The same pattern prevails for men with the use of body fat percentage (Table 4, Column 3).
} 
Table 2 BMI categories as determinants of SWB

\begin{tabular}{lllll}
\hline & $(1)$ & $(2)$ & $(3)$ & $(4)$ \\
& Women & Women & Men & -0.263 \\
\hline Underweight & 0.310 & 0.313 & -0.584 & $(0.615)$ \\
& $(0.351)$ & $(0.321)$ & $(0.681)$ & -0.0684 \\
Overweight & 0.0189 & 0.0479 & -0.0547 & $(0.0741)$ \\
& $(0.0763)$ & $(0.0680)$ & $(0.0848)$ & -0.177 \\
Obese & $-0.200^{*}$ & -0.00871 & $-0.340^{* * *}$ & $(0.111)$ \\
& $(0.104)$ & $(0.0929)$ & $(0.131)$ & $11.56 * * *$ \\
15D score & & $10.04 * * *$ & & $(0.858)$ \\
& & $(0.786)$ & 1,111 & 1,111 \\
$\mathrm{~N}$ & 1,134 & 1,134 & 0.024 & 0.254 \\
$\mathrm{R}^{2}$ & 0.015 & 0.228 & & \\
\hline
\end{tabular}

The baseline is normal weight. All models include the unreported controls: age and its square, education groups, and annual earnings. Estimation method is OLS. Robust standard errors in parentheses: *** $p<0.01, * * p<0.05, * p<0.1$

corresponding to no, moderate, or extreme problems. We used the most common tariff, the UK time-trade-off (TTO) values, to convert the states to health utility scores. EQ-5D TTO scores range from 1 (full health) to $-0.59(0=$ being dead). All the estimation results were otherwise similar except that the category for obese men obtained a statistically significant negative effect $(-0.2733)$ with a robust standard error of 0.1147 even after controlling for health status by using EQ-5D (not reported).

We have also estimated separate specifications for each component of 15D. Each health dimension is a statistically significant explanatory variable for SWB. Furthermore, almost all of the individual health dimensions contribute positively to the reduction in the impact of obesity on SWB. They are the following: mobility, hearing, breathing, sleeping, usual activities, discomfort and symptoms, depression, distress, vitality, and sexual activity. Therefore, the results are clearly not driven by any single component of health and functional capacity.

Because income is arguably an endogenous explanatory variable, we have tested whether the results are sensitive to its inclusion/exclusion. The conclusions remain the same when income is not included among the right-hand side variables (not reported).

Table 3 Waist circumference as a determinant of SWB

\begin{tabular}{lllll}
\hline & $(1)$ & $(2)$ & $(3)$ & $(4)$ \\
& Women & Women & Men & Men \\
\hline Waist circumference & $-0.00807^{* * *}$ & 0.000776 & $-0.00695^{*}$ & -0.00376 \\
& $(0.00306)$ & $(0.00273)$ & $(0.00400)$ & $(0.00335)$ \\
$15 \mathrm{D}$ score & & $10.20^{* * *}$ & & $11.64 * * *$ \\
& & $(0.788)$ & & $(0.858)$ \\
$\mathrm{N}$ & 1,122 & 1,122 & 1,109 & 1,109 \\
$\mathrm{R}^{2}$ & 0.016 & 0.231 & 0.018 & 0.254 \\
\hline
\end{tabular}

All models include the unreported controls: age and its square, education groups, and annual earnings. Estimation method is OLS. Robust standard errors in parentheses: *** $p<0.01$, ** $p<0.05$, * $p<0.1$ 
Table 4 Body fat percentage as a determinant of SWB

\begin{tabular}{lllll}
\hline & $(1)$ & $(2)$ & $(3)$ & $(4)$ \\
& Women & Women & Men & -0.00684 \\
\hline Fat mass $(\%)$ & $-0.0113^{* *}$ & -0.000988 & -0.0115 & $(0.00621)$ \\
& $(0.00544)$ & $(0.00471)$ & $(0.00726)$ & $11.73 * * *$ \\
$15 \mathrm{D}$ score & & $10.67 * * *$ & & $(0.907)$ \\
& & $(0.735)$ & 1,063 & 1,063 \\
$\mathrm{~N}$ & 1,063 & 1,063 & 0.015 & 0.252 \\
\hline
\end{tabular}

All models include the unreported controls: age and its square, education groups, and annual earnings. Estimation method is OLS. Robust standard errors in parentheses: *** $p<0.01$, ** $p<0.05, * p<0.1$

Table 5 Lean body mass as a determinant of SWB

\begin{tabular}{lllll}
\hline & $(1)$ & $(2)$ & $(3)$ & $(4)$ \\
& Women & Women & Men & Men \\
\hline Lean body mass & $-0.0111^{*}$ & $-3.16 \mathrm{e}-05$ & -0.00418 & -0.00406 \\
& $(0.00616)$ & $(0.00532)$ & $(0.00515)$ & $(0.00435)$ \\
$15 \mathrm{D}$ score & & $10.68^{* * *}$ & & $11.76^{* * *}$ \\
& & $(0.725)$ & & $(0.912)$ \\
$\mathrm{N}$ & 1,063 & 1,063 & 1,063 & 1,063 \\
$\mathrm{R}^{2}$ & 0.012 & 0.245 & 0.014 & 0.252 \\
\hline
\end{tabular}

All models include the unreported controls: age and its square, education groups, and annual earnings. Estimation method is OLS. Robust standard errors in parentheses: *** $p<0.01$, ** $p<0.05, * p<0.1$

Next, we present results from alternative estimators. To be effective, PSM should balance observable characteristics across the treatment and comparison groups in the region of common support. The quality of the match seems good; there are generally no statistically significant differences between the groups after matching (not reported). The ATT estimates in Table 6 based on PSM are almost similar to the ones obtained by using OLS in Table 2. Again, there is a significant reduction in the estimate after adding 15D to the set of variables. For women the effect of being obese on SWB is no longer statistically significant after adding 15D. However, for men the effect of being obese on SWB is statistically significant at the $10 \%$ level even though the point estimate is considerably lower with 15D added (Column 4 of Table 6). Therefore, for women PSM confirms our

Table 6 Propensity score estimates for the effect of being obese on SWB

\begin{tabular}{lllll}
\hline & $(1)$ & $(2)$ & $(3)$ & $(4)$ \\
& Women & Women & Men & Men \\
\hline \multirow{2}{*}{ ATT } & $-0.214^{* *}$ & -0.0684 & $-0.3114^{* * *}$ & $-0.1497^{*}$ \\
\multirow{2}{*}{$15 \mathrm{D}$ score } & $(0.0827)$ & $(0.0754)$ & $(0.0843)$ & $(0.0774)$ \\
& Excluded & Included & Excluded & Included \\
\hline
\end{tabular}

The table reports ATTs. They are calculated using Kernel matching (Epanechnikov) and matching is performed using the region of common support for the propensity scores. Caliper is set at 0.001. Bootstrap standard errors for ATTs (1,000 replications) in parentheses: *** $p<0.01, * * p<0.05, * p<0.1$ 
earlier finding. The ATT for men with 15D added is lower than the corresponding OLS estimate in Column 4 of Table 2. The main difference in the results is that the standard error for the ATT obtained by using bootstrapping is much lower in Table 6 .

Lastly, we have also experimented with height as an instrument for BMI, following Katsaiti (2012). The validity of the IV approach is not dependent on CIA (Conditional Independence Assumption) as previous OLS and matching estimates. The idea for the use of height as an instrument for BMI is that well-being shocks cannot have an effect on height, because height is a predetermined variable for individuals aged 30 or more. However, there is a non-linear relationship between BMI and height, by definition. The 1st stage of this IV approach works well for women. (The F-test statistic is 14.49.) We used the same control variables as in all other models (e.g. including annual earnings). ${ }^{9}$ These results reveal that BMI is not a statistically significant determinant of SWB in the IV specifications for women, not even without adding 15D to the set of controls (not reported). But the IV estimates have larger standard errors than the OLS estimates, as expected. Furthermore, we also experimented with using the square of height as an instrument for BMI. But it turned out that this does not alter our results. Taken together, it is fair to say that the relatively small sample size limits the use of the IV approach.

\section{Conclusions}

In this paper we find further evidence of the already well-known fact that obesity is associated with lower subjective well-being. More importantly, we find that this generally holds regardless of what kind of overweight or body mass measure we use. This is useful, because we know from previous research that BMI alone does not fully capture the adverse health effects of obesity (Pischon et al. 2008; Petursson et al. 2011). The main message of this paper, however, is that, when controlling properly for health and functional capacity, the negative association between obesity and SWB disappears almost completely. Thus, it is not obesity itself that is the problem for individual SWB, but the health and functional capacity problems it brings. In other words, being overweight seems to be acceptable until it decreases health or functionality, at the least in the Finnish context.

But for men the results from some of the robustness checks give a slightly more mixed picture. We found some evidence of a decrease in SWB even after controlling for health and functional capacity. It is also interesting to note that for women we find that lean body mass is associated with a decrease in the level of SWB without a control for health and functional status. One plausible explanation for this finding is that for men having a lot of lean body mass is socially acceptable.

The main strength of this study is the use of a health-related quality-of-life measure (i.e. 15D) that captures the concept of health as wholly as possible. Another important strength is the use of large, representative, general population data with a high response rate, allowing the precise estimation of the effects as they are experienced in the population on average. This contrasts with clinical studies where patients are somehow selected and usually more severely ill. The most important limitations of our study are the facts that we used only one measure of SWB due to the shortcomings of the Health 2000 Survey and that we were not able to address causal effects in the baseline specifications. Also, owing to the limitations of our data we were unable to include all the factors that might mitigate the

\footnotetext{
${ }_{9}$ Katsaiti (2012, p. 4109) points out that it is important to control for income, because studies have shown that height is not connected to subjective well-being after controlling for income level.
} 
relationship between obesity and SWB. For example, wealth is not observed in our data. Obese but wealthy persons may be more functional and obesity might cause fewer losses to their SWB, other things being equal. Therefore, an obese person with a substantial amount of wealth is, for example, able to use his or her own car for mobility rather than public transport, where obesity evidently creates more limits to daily activities.

In this study we focused on persons aged 30 and over. It is possible that the estimated relationships are different for different groups of people, which requires further study. In addition, there is an apparent need to estimate the relationships between obesity and subjective well-being in other cultural contexts. This is important, because the strength of social stigma associated with obesity is most likely not universal but depends on e.g. the prevalence of obesity and social conventions. High prevalence of obesity in society may lessen the social stigma associated with obesity. Furthermore, there will be an obvious need to work with longitudinal data on this and related research questions in the future. With that kind of data, it will be possible to explore changes in various measures in obesity and functional capacity, and relate them to the changes in SWB. Thus, we will be in a much better position to say something about the exact causal pathways between obesity and SWB.

Acknowledgments We are grateful to anonymous referees for valuable comments that have greatly improved the paper. Paul A. Dillingham has kindly checked the English language.

\section{References}

Aromaa, A., \& Koskinen, S. (2004). (Eds). Health and functional capacity in Finland. Baseline results of the Health 2000 health examination survey. Helsinki: National Public Health Institute.

Audretsch, D. B., \& DiOrio, D. (2007). The spread of obesity. In Z. J. Acs \& A. Lyle (Eds.), Obesity, business and public policy (pp. 15-24). Cheltenham: Edward Elgar Publishing Limited.

Böckerman, P., \& Ilmakunnas, P. (2009). Unemployment and self-assessed health: Evidence from panel data. Health Economics, 18, 161-179.

Bozoyan, C., \& Wolbring, T. (2011). Fat, muscles, and wages. Economics and Human Biology, 9, 356-363.

Burkhauser, R. V., \& Cawley, J. (2008). Beyond BMI: The value of more accurate measures of fatness and obesity in social science research. Journal of Health Economics, 27, 519-529.

Cai, L., Lubitz, J., Flegal, K. M., \& Pamuk, E. (2010). The predicted effects of chronic obesity in middle age on Medicare costs and mortality. Medical Care, 48, 510-517.

Cawley, J. (2007). The labor market impact of obesity. In Z. J. Acs \& A. Lyle (Eds.), Obesity, business and public policy (pp. 76-86). Cheltenham: Edward Elgar Publishing Limited.

Cawley, J., \& Ruhm, C. J. (2012). The economics of risky health behaviors. In T. G. McGuire, M. V. Pauly, \& P. P. Barros (Eds.), Handbook of health economics (Vol. 2, pp. 95-199). New York: Elsevier.

Clark, A. E., \& Etilé, F. (2009). Happy house: Spousal weight and individual well-being. Journal of Health Economics, 30, 1124-1136.

Diener, E., Emmons, R. A., Larsen, R. J., \& Griffin, S. (1985). The satisfaction with life scale. Journal of Personality Assessment, 49, 71-75.

Diener, E., Suh, E. M., Lucas, R. E., \& Smith, H. L. (1999). Subjective well-being: Three decades of progress. Psychological Bulletin, 125, 276-302.

Dolan, P., Layard, R., \& Metcalfe, R. (2011). Measuring subjective wellbeing for public policy: Recommendations on measures. London: London School of Economics, Centre for Economic Performance, Special Paper No. 23.

Flegal, K. M., Carroll, M. D., Kit, B. K., \& Ogden, C. L. (2012). Prevalence of obesity and trends in the distribution of body mass index among US Adults, 1999-2010. Journal of the American Medical Association, 307, 491-497.

Flegal, K. M., Graubard, B. I., Williamson, D. F., et al. (2005). Excess deaths associated with underweight, overweight, and obesity. Journal of the American Medical Association, 293, 1861-1867.

Foster, R., \& Moore, E. (2012). Adolescent obesity and life satisfaction: Perceptions of self, peers, family, and school. Economics and Human Biology, 10, 385-394. 
Garratt, A., Schmidt, L., Mackintosh, A., \& Fitzpatrick, R. (2002). Quality of life measurement: Bibliographic study of patient-assessed health outcome measures. British Medical Journal, 324, 1417.

Heckman, J., Ichimura, H., Smith, J., \& Todd, P. (1998). Characterizing selection bias using experimental data. Econometrica, 66, 1017-1098.

Heistaro, S. (2008). (Ed). Methodology report. Health 2000 survey. Helsinki: National Public Health Institute.

Johansson, E., Böckerman, P., Kiiskinen, U., \& Heliövaara, M. (2009). Obesity and labour market success in Finland: The difference between having a high BMI and being fat. Economics and Human Biology, 7 , 36-45.

Katsaiti, M.-S. (2012). Obesity and happiness. Applied Economics, 44, 4101-4114.

Kolotkin, R. L., Meter, K., \& Williams, G. R. (2001). Quality of life and obesity. Obesity Reviews, 2, 219-229.

Kushner, R. F., \& Foster, G. D. (2000). Obesity and quality of life. Nutrition, 16, 947-952.

Lahti-Koski, M., Seppänen-Nuijten, E., Männistö, S., Härkänen, T., Rissanen, H., Knekt, P., et al. (2010). Twenty-year changes in the prevalence of obesity among Finnish adults. Obesity Review, 11, 171-176.

Larsson, U., Karlsson, J., \& Sullivan, M. (2002). Impact of overweight and obesity on health-related quality of life-a Swedish population study. International Journal of Obesity and Related Metabolic Disorders, 26, 417-424.

Linna, M. S., Kaprio, J., Raevuori, A., Sihvola, E., Keski-Rahkonen, A., \& Rissanen, A. (2013). Body mass index and subjective well-being in young adults: A twin population survey. BMC Public Health, 13, 231.

Morris, S. (2007). The impact of obesity on employment. Labour Economics, 14, 413-433.

OECD. (2010). Obesity and the economics of prevention: Fit not fat. Paris: OECD.

Oswald, A. (1997). Happiness and economic performance. Economic Journal, 107, 1815-1831.

Oswald, A. (2010). Emotional prosperity and the Stiglitz commission. British Journal of Industrial Relations, 48, 651-669.

Oswald, A., \& Powdthavee, N. (2007). Obesity, unhappiness, and the challenge of affluence: Theory and evidence. Economic Journal, 117, F441-F459.

Petursson, H., Sigurdsson, J. A., Bengtsson, C., Nilsen, T. I., \& Getz, L. (2011). Body configuration as a predictor of mortality: Comparison of five anthropometric measures in a 12 year follow-up of the Norwegian HUNT 2 study. PLos One, 6, 10.

Pischon, T., Boeing, H., Hoffmann, K., et al. (2008). General and abdominal adiposity and risk of death in Europe. New England Journal of Medicine, 359, 2105-2120.

Scharfetter, H., Schlager, T., Stollberger, R., Felsberger, R., Hutten, H., \& Hinghofer-Szalkay, H. (2004). Assessing abdominal fatness with local bioimpedance analysis: Basics and experimental findings. International Journal of Obesity, 25, 502-511.

Sintonen, H. (2001). The 15D instrument of health-related quality of life: Properties and applications. Annals of Medicine, 33, 328-336.

Stutzer, A. (2007). Limited self-control, obesity and the loss of happiness. Bonn: IZA Discussion Paper No. 2925.

The EuroQoL Group. (1990). EuroQolda-new facility for the measurement of health-related quality of life. Health Policy, 16, 199-208.

Tinkler, L., \& Hicks, S. (2011). Measuring subjective well-being. London: Office for National Statistics.

Wada, R., \& Tekin, E. (2010). Body composition and wages. Economics and Human Biology, 8, $242-254$.

Watson, D., Clark, L. A., \& Tellegen, A. (1988). Development and validation of brief measures of positive and negative affect: The PANAS Scales. Journal of Personality and Social Psychology, 54, $1063-1070$.

WHO (2000). Obesity: Preventing and managing the global epidemic. Technical report series No. 894. World Health Organization, Geneva. 